Bull. Fac.Agric.,Cairo Univ., 63: 450-461(2012).

\title{
LAND EVALUATION FOR SUSTAINABLE PRODUCTIVITY IN THE SOUTHERN TOHAMA PLAINS, SAUDI ARABIA
}

(Received:21.10.2012)

\author{
By \\ A. S.Al-Farraj, A. Sh. Sallam, A. M. Al-Turki, A. S. Al-Malik and G. Abdel-Nasser \\ Soil Science Department, College of Food Sciences and Agriculture, \\ King Saud University, Saudi Arabia
}

\begin{abstract}
To utilize the precious water resources most efficiently, land has to be selected for irrigation according to its suitability for agricultural production. Therefore, the southern Tohama plains were selected to evaluate the soils of the main landforms for sustainable agricultural production using qualitative and quantitative evaluation methods, according to type, number and degree of agriculture limitations such as; texture, effective soil depth, salinity, gravels and calcium carbonate content..etc. This study was carried out in 2004-2008 as part of a project aimed to "Survey of soil resources and water quality evaluation in Southern Tohama plains, Saudi Arabia". The Tohama plains have important geomorphologic features in the south western region of Saudi Arabia, which receive relatively high quantities of runoff water from the surrounding hills. Soils of Southern Tohama plains are the most important source of available land resources for sustainable agriculture development in the region. Four hundred eighty four soil profiles representing the main landforms were examined. Soil characteristics were rated for different systems of irrigated agriculture according to the recommended methods. Generally, the unsuitable soils for agriculture ( $\mathrm{N} 1$ and $\mathrm{N} 2$ ) occupied varying areas in the dominant landforms in the study area as follows: Sabkhat (61\%), Coastal plains (20\%), Wadis (22\%) and Footslopes (23\%). While, the different suitable classes for irrigated agriculture occupied most of the dominant landforms such as alluvial plain, alluvial fans ...etc. Also, the results showed that the high suitable soils represent $13 \%$ of total area, while most of the area includes suitable soils with restriction (36\% of total area). The unsuitable soils represent less area $(2.2 \%)$.
\end{abstract}

Key words: landforms, land suitability, Saudi Arabia, Tohama plains.

\section{INTRODUCTION}

Considering the rapid growth of the world populations, which is in its turn a limiting factor to the arable lands around the world, the dire need for effective and efficient application of the croplands has been felt more than ever. Sustainable agriculture would be achieved if lands can be categorized and utilized based upon their different uses (FAO, 1983, 1984).

Problems caused by immethodical and unsuitable using of lands, irregular development of urban areas and therefore reduction in cultivation zones have received widespread attention throughout the world. Using of optimum and suitable methods of lands is the main concern of policy makers for the sustainable planning and management most of the cultivation areas. Hence, studies related to land suitability evaluation will provide sustainable using agricultural lands (Rahimi et al., 2009). The capacity of land to produce plants is subjected to some limitation. The limits for production are set by soil, landform, water supply and climate conditions and the use and management applied to the land. Accordingly, knowledge of land resource endowments and their production potential is an essential prerequisite for planning optimal land use in changeable socioeconomic scenarios. This knowledge is equally important to effect the desired change i.e. agricultural and economic development that could be sustained for a reasonably long time (FAO, 1993).

The fundamental principle of land evaluation is to estimate the potential of a land for different productive uses, such as farming, livestock production, or forestry, together with uses that provide services or other benefits, such as water catchment, recreation, tourism and wildlife conservation (Dent and Young, 1981). Consequently, land evaluation is a tool for 
strategic land use planning. A specific agricultural use and management system on land that is most suitable according to agro-ecological potentialities and limitations is the best way to achieve sustainability (FAO, 1978).

The most widely used approaches for suitability classification for a particular use are discussed using FAO's framework for land evaluation (FAO, 1976) and Sys (1979). The basic data used in both systems for evaluating land suitability for specific crops and the kind of irrigation and management levels include topography, soils, water and other features such as infrastructure, markets and socio-economic conditions. In the Kingdom of Saudi Arabia several studies were carried out to classify soils (Mashhady et al., 1986; Ministry of Agriculture and Water 1985 and 1995; Al-Malik, 1994 and Al-Sheikh et al., 1995).

The suitability of a given piece of land is its natural ability to support a specific purpose. According to the FAO methodology (1976), this is strongly related to the "land qualities" such as erosion resistance, water availability, and flood hazard that are not measurable. As these qualities derive from the "land characteristics", such as slope angle and length, rainfall and soil texture which are measurable or estimable, it is advantageous to use these later values to study the suitability. Thus, the land characteristic parameters were used to workout land suitability for irrigation, crops and forest. The land suitability classification consists of assessing and grouping the land types in orders and classes according to their aptitude. Sys et al. (1991) applied the concept of FAO and assessed the land in irrigated and rainfed area. Data on crop requirements are also provided (Sys et al., 1993).

The western coastal lowlands of Saudi Arabia, locally called Tohama, form a narrow strip along the Red Sea coast. In the north, it starts as a narrow plain near Al Wajh and gradually broadens to more than $40 \mathrm{Km}$ near Gizan in the south. The Tohama is low depositional surface mostly on a coral plain that grades upwards to the east to form a pediment on hard crystalline rock of the geologic basement. Along its eastern edge the coastal plain is set apart from the highlands by an escarpment wall, which runs parallel to the sea. The landforms, developed in this region, are mainly of alluvial nature, formed as a result of the downward transportation of soil material from the highlands by the many wadis and drainage channels that drain out in the sea. The watercourses are gently sloping and short, with the exception of wadi al Hamd, near Madinah, which extends inland to a long distance. Landforms in the forms of alluvial fans, alluvial plains, deltas or forms of footslopes of colluvial nature are common. Along the shore, tidal flats and beaches are developed by tidal activities of the sea (Ministry of Agriculture and Water, 1995). General survey was conducted on the soil of Saudi Arabia (Ministry of Agriculture and Water, 1995), the report included an assessment for the land resources. Also, the report pointed out that each land resource unit is evaluated for its suitability for general cropping and 33 specific crops under two relevant modes of irrigation, i.e. surface or overhead means. In high rainfall areas, such as terraced slopes in the Asir mountains, land potential for rainfed cultivation is also assessed. For irrigation, land suitability for the crops is determined with various assumptions about groundwater, including its availability (or otherwise) and diverse qualities of the water.

The main objective of the current study was to evaluate the soils of the main landforms of Tohama plains in the southwestern region of Saudi Arabia for sustainable agricultural production using qualitative and quantitative evaluation methods, according to type, number and degree of agriculture limitations such as; texture, effective soil depth, salinity, gravels and calcium carbonate content..etc. This appraisal is designed to assist the development planners in identifying and assessing areas suitable for agricultural production.

\section{MATERIALS AND METHODS 2.1. Description of the study area}

Tohama plains are one of the more promising areas in the Kingdom for future sustainable agriculture development, where the requirements for sustainability exist including renewable surface water resources and deep fertile soils. In addition, the region is one of the oldest agriculture areas in Saudi Arabia with accumulated experience for successfully cultivating field crops, vegetables and fruit trees. The region has worm summer and temperate winter that are suitable for promising agriculture development for traditional and intensive cultivations. The relatively high annual rainfalls, particularly in the surrounding mountains are the main renewable water resource that causing occasion flooding in the wadis and plains in Tohama. The study area is located between the international boarders of Saudi Arabia with Yemen (Latitude $16^{\circ} 24^{\prime} 26^{\prime \prime} \mathrm{N}$ ) and north Wadi Rim $\left(17^{\circ} 48^{\prime} 29.9^{\prime \prime} \mathrm{N}\right)$ with a length of 
$170 \mathrm{Km}$ and a mean width of $30 \mathrm{Km}$, the total area is $8165 \mathrm{Km}^{2}$ (Fig. 1). The area was occupied by weathered products of igneous and metamorphic rocks. These rocks could be grouped into three formations; granite, basalt and schist and they constitute the main source of soil parent materials. The meteorological data (Ministry of Agriculture and Water, 1988) indicated that the annual average rainfall, temperature and relative humidity were $100-200 \mathrm{~mm} /$ year, $21-40{ }^{\circ} \mathrm{C}$ and $85 \%$, respectively. The main source of irrigation water is the underground water from aquifer. Generally, the total soluble salts of the ground water have high water quality $\left(\mathrm{EC}_{\mathrm{iw}}<3.0 \mathrm{dSm}^{-1}\right)$ (Al-Turki et al., 2008).

\subsection{Field study and laboratory analyses}

Soil properties (morphological, physical and chemical), water characteristics and climatological data of the study area were derived from a semidetailed soil study regarding Tohama plains. The total soil profiles were accounted as 484 profiles, representing twelve geomorphic units encountered in the study area. (Al-Turki et al., 2008). The main objective of this study was to survey of land resources and evaluation of water quality in southern Tohama plain (Al-Turki et al., 2008).

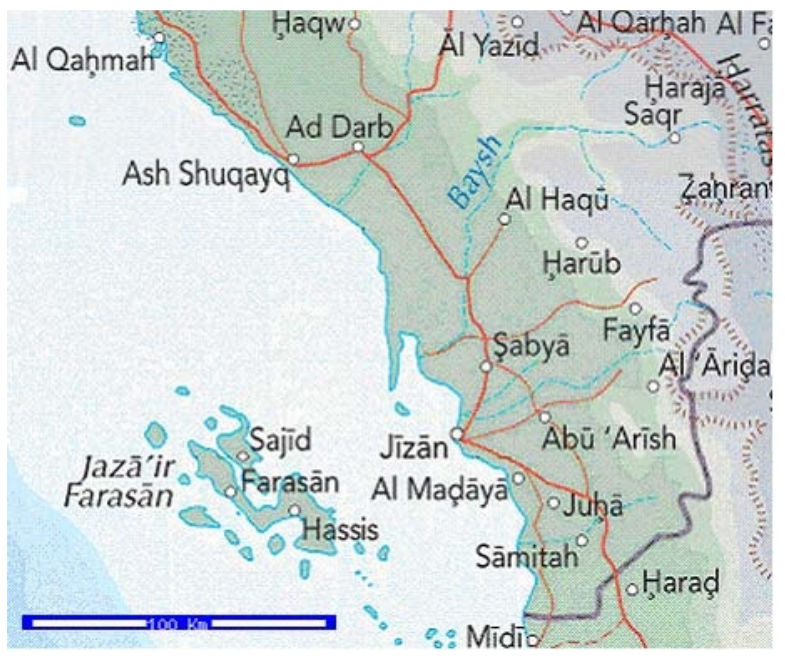

Fig. (1). Location of the study area.

These soil characteristics were rated for irrigated agriculture according to Sys and Verheye (1978), different systems of irrigation according to FAO (1979 and 1983). The land evaluation was determined based upon topography (t), Depth (s2) (s2'= for bedrock, s2 for water table), Drainage $(\mathrm{w})$, Texture (s1).(s1'=0 $-25 \mathrm{~cm}, \mathrm{~s} 1=>25 \mathrm{~cm}$ depth), Coarse fragments (s4) (s4'=0-25cm, S4= $>25 \mathrm{~cm}$ depth), Salinity \& Alkalinity (n), and $\mathrm{CaCO}_{3} \%$ ( s3). The topography characteristics included slope, while soil properties included soil texture, depth, salinity, drainage and carbonate content. Also, soil properties such as Cation Exchange Capacity (CEC), Percentage of Basic Saturation (PBS), Organic matter (\%OM) and $\mathrm{pH}$ were considered in terms of soil fertility. Sys et al. (1991) suggested that soil characteristics such as $\% \mathrm{OM}$ and $\mathrm{PBS}$ do not require any evaluation in the arid regions while clay CEC rate usually exceeds the plant requirements without further limitation, thus, fertility properties can be excluded from land evaluation with the purpose of irrigation.

\section{RESULTS AND DISCUSSIONS}

The two systems of land evaluation were tested in this study, they are:

- The parametric evaluation system for irrigated agriculture according to Sys and Verheye (1978).

- The qualitative evaluation system for irrigation according to FAO (1979 and 1983).

The two classification systems were based on FAO framework categorical levels; i.e.:

1- Land suitability order $(\mathrm{S}, \mathrm{N})$; reflecting kind of suitability.

2- Land suitability classes (S1, S2, S3, N1, N2); reflecting degrees of suitability.

3- Land suitability subclasses ( $\left.\mathrm{S} 2_{(. .)}\right)$; reflecting kinds of limitations or main kinds of improvement measures required within classes.

4- Land suitability units; reflecting minor differences in required management within subclasses.

The evaluation relies on standard land characteristics including environmental, physical, chemical and fertility soil characteristics as follows:

1- Environmental factors; climate (c), topography

$(\mathrm{t})$ and drainage $(\mathrm{w})$.

2- Physical soil characteristics (s); texture / structure (s1), effective soil depth (s2), carbonate content (s3) and rock fragments (s4).

3- Chemical soil characteristics (n); EC and ESP.

4- Fertility soil characteristics (f); $\mathrm{pH}$ (f1), organic matter (f2), sum of basic cations (f3) and apparent CEC (f4).

3.1. The parametric evaluation system according to Sys and Verheye (1978)

This system aims to evaluate land suitability for irrigated agriculture. The different land characteristics that influence the soil suitability for irrigation are rated and capability index for irrigation $(\mathrm{Ci})$ is calculated according to the formula: 
$\mathrm{Ci}=\mathrm{t} . \mathrm{w} / 100$. s1/100. s2/100. s3/100. s4/100. $\mathrm{n} / 100$

The capability classes are defined according to the intensity of soil limitations and the value of the capability index (Ci), Table (1). content of the top $30 \mathrm{~cm}$ is less than $50 \%$. If higher the weighted average of the upper $30 \mathrm{~cm}$ is considered only.

The evaluation of land characteristics can be achieved in a relative limitation scale where five

Table (1): Criteria for the determination of the land suitability classes.

\begin{tabular}{|l|l|l|}
\hline \multicolumn{1}{|c|}{ Class } & Capability index $(\mathbf{C i})$ & \multicolumn{1}{c|}{ Criteria } \\
\hline S1: Very suitable & $>75$ & Land units with no or only 3/4 slight limitations. \\
\hline $\begin{array}{l}\text { S2:Moderately } \\
\text { suitable }\end{array}$ & $\mathbf{5 0 - 7 5}$ & $\begin{array}{l}\text { Land units with slight and no more than } 2 / 3 \text { moderate } \\
\text { limitations. }\end{array}$ \\
\hline $\begin{array}{l}\text { S3 Marginally } \\
\text { suitable }\end{array}$ & $\mathbf{2 5 - 5 0}$ & $\begin{array}{l}\text { Land units with more than } 2 / 3 \text { moderate limitations and no } \\
\text { more than 1 severe limitation that however doesn't } \\
\text { excludes the use of the land. }\end{array}$ \\
\hline N1: Not suitable & $<25$ & Land units with severe limitation that can be corrected. \\
\hline N2: Not suitable & $<25$ & $\begin{array}{l}\text { Land units with severe and /or very severe limitation that } \\
\text { can't be corrected. }\end{array}$ \\
\hline
\end{tabular}

In this system, the land characteristics to be used for evaluation are:

- topography (t)

- wetness (w):

*flooding

*drainage

- physical soil characteristics:

Texture, effective soil depth, calcium carbonate content, and salinity and alkalinity.

The dominant topographic factor that influences the irrigation suitability, concerns the slope. Wetness limitations are evaluated with regard to drainage conditions and flooding. The criteria for the evaluation of drainage conditions are without or with presence of saline groundwater table. The textural evaluation of the profile is done to the depth of $120 \mathrm{~cm}$, considering that the surface horizon is more important than the subsurface ones. Soil depth is defined as the thickness of the loose soil above a limited layer, which is impermeable for roots or percolating water. The most common types of such limiting layers are:

- an unconsolidation gravelly or stony horizon with at least $75 \%$ coarse fragments.

- a continuous, more or less consolidated, calcium carbonate or gypsiferous layer with a minimum thickness of $30 \mathrm{~cm}$ and including at least $50 \%$ $\mathrm{CaCO}_{3}$ or gypsum.

- a continuous hard rock or hardpan of more than $10 \mathrm{~cm}$ thick.

The criteria to evaluate $\mathrm{CaCO}_{3}$ status depend on the weighted average of the profile until the depth of $1 \mathrm{~m}$ or up to a limited layer if the $\mathrm{CaCO}_{3}$ levels are used, Table (2).

Table (2): Limitation levels and their rating.

\begin{tabular}{|c|c|c|}
\hline Symbol & Intensity of limitation & \multicolumn{1}{|c|}{ Rating } \\
\hline $\mathbf{0}$ & No & $\mathbf{9 8 - 1 0 0}$ \\
\hline 1 & Slight & $\mathbf{8 5}-\mathbf{9 8}$ \\
\hline 2 & Moderate & $\mathbf{6 0 - 8 5}$ \\
\hline 3 & Severe & $\mathbf{4 5}-\mathbf{6 0}$ \\
\hline 4 & Very severe & $<\mathbf{4 5}$ \\
\hline
\end{tabular}

Data in Table (3) indicate the different subclasses that could be defined in the soils of the study area according to Sys and Verheye (1978). Due to the large number of soil profiles that represents the dominant landforms in the study area, the study only included a number of soil profiles representing different degrees of suitability within each form of land. While the rest of the soil profiles covered by the current study are located in the report of Al-Turki et al., 2008.

According to (Sys and Verhey 1978) the percentage of studied soil profile belonging to different suitability classes within each landform (of the total studied soil profile represent every landform) was as follows: highly suitable land (S1) land within this class is quite limited (alluvial plain $22 \%$, pediplain with shallow soil $28 \%$, hills and rock outcrops $18 \%$, footslopes $19 \%$, terraced $11 \%$, and alluvial fan $18 \%$ ). Moderately suitable land (S2) lands within this suitability class have moderate limitations (coastal plain 11\%, alluvial plain $34 \%$, wadis $27 \%$, pediplain with deep soil $51 \%$, pediplain with shallow soil $28 \%$, active 
Table (3): Land Suitability for Irrigated Agriculture According to (Sys and Verhey 1978).

\begin{tabular}{|c|c|c|c|c|c|c|c|c|c|}
\hline Profile No. & $\mathbf{t}$ & $\mathbf{w}$ & s1 & s2 & s3 & s4 & $\mathbf{n}$ & C1 & Subclass \\
\hline \multicolumn{10}{|c|}{ Sabkhat } \\
\hline 1 & $0_{(100)}$ & $3_{(55)}$ & $2_{(78)}$ & $3_{(55)}$ & $0_{(100)}$ & $\mathrm{S} 1_{(90)}$ & $4_{(45)}$ & 9.6 & $\mathrm{~N} 1_{(\mathrm{n}, \mathrm{w})}$ \\
\hline 47 & $0_{(100)}$ & $\mathrm{S} 1_{(95)}$ & $2_{(70)}$ & $0_{(100)}$ & $0_{(100)}$ & $0_{(100)}$ & $3_{(58)}$ & 38.6 & $\mathrm{~S} 3_{(\mathrm{s} 1, \mathrm{n})}$ \\
\hline 48 & $0_{(100)}$ & $2_{(70)}$ & $2_{(77)}$ & $\mathrm{S} 1_{(90)}$ & $0_{(100)}$ & $0_{(100)}$ & $2_{(75)}$ & 36.4 & $\mathrm{~S} 3_{(\mathrm{s} 1, \mathrm{n}, \mathrm{w})}$ \\
\hline 63 & $\mathrm{~S} 1_{(90)}$ & $2_{(70)}$ & $3_{(53)}$ & $\mathrm{S} 1_{(95)}$ & $0_{(100)}$ & $0_{(100)}$ & $0_{(100)}$ & 23.5 & $\mathrm{~S} 3_{(\mathrm{s} 1, \mathrm{w})}$ \\
\hline 166 & $0_{(100)}$ & $3_{(55)}$ & $S 1_{(90)}$ & $2_{(70)}$ & $0_{(100)}$ & $0_{(100)}$ & $2_{(85)}$ & 29.5 & $\mathrm{~S} 3_{(\mathrm{w}, \mathrm{n}, \mathrm{s} 2)}$ \\
\hline 194 & $0_{(100)}$ & $3_{(55)}$ & $4_{(30)}$ & $2_{(70)}$ & $\mathrm{S} 1_{(90)}$ & $0_{(100)}$ & $2_{(85)}$ & 8.8 & $\mathrm{~N} 1_{(\mathrm{w}, \mathrm{s} 1, \mathrm{n}, \mathrm{s} 2)}$ \\
\hline \multicolumn{10}{|c|}{ Coastal plain } \\
\hline 2 & $2_{(85)}$ & $2_{(80)}$ & $3_{(45)}$ & $0_{(100)}$ & $0_{(100)}$ & $0_{(100)}$ & $0_{(100)}$ & 30.6 & $\mathrm{~S} 3_{(\mathrm{si}, \mathrm{w}, \mathrm{t})}$ \\
\hline 17 & $3_{(60)}$ & $\mathrm{S} 1_{(86)}$ & $2_{(69)}$ & $0_{(100)}$ & $0_{(100)}$ & $0_{(100)}$ & $\mathrm{S} 1_{(90)}$ & 31.7 & $\mathrm{~S} 3_{(\mathrm{t}, \mathrm{s} 1)}$ \\
\hline 24 & $0_{(100)}$ & $\mathrm{S} 1_{(95)}$ & $S 1_{(93)}$ & $0_{(100)}$ & $0_{(100)}$ & $0_{(100)}$ & $3_{(45)}$ & 39.8 & $\mathrm{~S} 3_{(\mathrm{n})}$ \\
\hline 46 & $\mathrm{~S} 1_{(90)}$ & $3_{(45)}$ & $3_{(52)}$ & $0_{(100)}$ & $0_{(100)}$ & $0_{(100)}$ & $\mathrm{S} 1_{(90)}$ & 19 & $\mathrm{~N} 1_{(\mathrm{w}, \mathrm{s} 1)}$ \\
\hline 49 & $S 1_{(90)}$ & $3_{(45)}$ & $S 1_{(91)}$ & $0_{(100)}$ & $0_{(100)}$ & $0_{(100)}$ & $0_{(98)}$ & 36.1 & $\mathrm{~S} 3_{(\mathrm{w})}$ \\
\hline 180 & $0_{(100)}$ & $3_{(45)}$ & $2_{(82)}$ & $0_{(100)}$ & $0_{(100)}$ & $0_{(100)}$ & $3_{(58)}$ & 21.4 & $\mathrm{N1} 1_{(\mathrm{w}, \mathrm{n})}$ \\
\hline 188 & $0_{(100)}$ & $0_{(100)}$ & $2_{(75)}$ & $0_{(100)}$ & $0_{(100)}$ & $0_{(100)}$ & $0_{(100)}$ & 75 & $\mathrm{~S} 2_{(\mathrm{s} 1)}$ \\
\hline 216 & $0_{(100)}$ & $2_{(68)}$ & $\mathrm{S1}_{(93)}$ & $0_{(100)}$ & $0_{(100)}$ & $0_{(100)}$ & $0_{(100)}$ & 63.2 & $\mathrm{~S} 2_{(\mathrm{w})}$ \\
\hline \multicolumn{10}{|c|}{ Wadis } \\
\hline 3 & $0_{(100)}$ & $2_{(60)}$ & $3_{(55)}$ & $0_{(100)}$ & $0_{(100)}$ & $0_{(100)}$ & $0_{(100)}$ & 33 & $\mathrm{~S} 3_{(\mathrm{s} 1, \mathrm{w})}$ \\
\hline 25 & $0_{(100)}$ & $\mathrm{S} 1_{(95)}$ & $2_{(80)}$ & $0_{(100)}$ & $0_{(100)}$ & $0_{(100)}$ & $0_{(100)}$ & 75 & $\mathrm{~S} 2_{(\mathrm{s} 1)}$ \\
\hline 30 & $0_{(100)}$ & $0_{(100)}$ & $3_{(45)}$ & $0_{(100)}$ & $0_{(100)}$ & $0_{(100)}$ & $0_{(100)}$ & 45 & $\mathrm{~S} 3_{(\mathrm{s} 1)}$ \\
\hline 133 & $0_{(100)}$ & $0_{(100)}$ & $S 1_{(90)}$ & $0_{(100)}$ & $0_{(100)}$ & $0_{(100)}$ & $0_{(100)}$ & 90 & S1 \\
\hline 134 & $0_{(100)}$ & $0_{(100)}$ & $3_{(55)}$ & $2_{(75)}$ & $0_{(100)}$ & $0_{(100)}$ & $0_{(98)}$ & 40.4 & $\mathrm{~S} 3_{(\mathrm{s} 1, \mathrm{~s} 2)}$ \\
\hline 162 & $0_{(100)}$ & $3_{(45)}$ & $4_{(40)}$ & $0_{(100)}$ & $0_{(100)}$ & $0_{(100)}$ & $0_{(100)}$ & 18 & $\mathrm{~N} 1_{(\mathrm{W}, \mathrm{s} 1)}$ \\
\hline 191 & $0_{(100)}$ & $0_{(100)}$ & $0_{(100)}$ & $2_{(75)}$ & $0_{(100)}$ & $0_{(100)}$ & $0_{(100)}$ & 75 & $\mathrm{~S} 2_{(\mathrm{s} 2)}$ \\
\hline 199 & $3_{(50)}$ & $3_{(45)}$ & $3_{(55)}$ & $3_{(55)}$ & $0_{(100)}$ & $0_{(100)}$ & $0_{(100)}$ & 6.8 & $\mathrm{~N} 2$ \\
\hline 209 & $0_{(100)}$ & $3_{(45)}$ & $3_{(53)}$ & $3_{(55)}$ & $0_{(100)}$ & $0_{(100)}$ & $0_{(100)}$ & 13.1 & $\mathrm{~N} 1_{(\mathrm{s} 2, \mathrm{~s} 1)}$ \\
\hline 219 & $0_{(100)}$ & $3_{(45)}$ & $4_{(30)}$ & $3_{(55)}$ & $1_{(90)}$ & $0_{(100)}$ & $0_{(100)}$ & 6.7 & $\mathrm{~N} 2$ \\
\hline \multicolumn{10}{|c|}{ Alluvial plain } \\
\hline 26 & $0_{(100)}$ & $22_{(62)}$ & $2_{(82)}$ & $0_{(100)}$ & $0_{(100)}$ & $0_{(100)}$ & $0_{(100)}$ & 50.8 & $\mathrm{~S} 2_{(\mathrm{s} 1, \mathrm{w})}$ \\
\hline 27 & $0_{(100)}$ & $2_{(60)}$ & $0_{(98)}$ & $0_{(100)}$ & $0_{(100)}$ & $0_{(100)}$ & $0_{(100)}$ & 58.8 & $\mathrm{~S} 2_{(\mathrm{w})}$ \\
\hline 28 & $0_{(100)}$ & $0_{(100)}$ & $3_{(50)}$ & $0_{(100)}$ & $0_{(100)}$ & $0_{(100)}$ & $0_{(100)}$ & 50.0 & $\mathrm{~S} 3_{(\mathrm{s} 1)}$ \\
\hline 31 & $0_{(100)}$ & $0_{(100)}$ & $1_{(96)}$ & $0_{(100)}$ & $0_{(100)}$ & $0_{(100)}$ & $0_{(100)}$ & 96 & S1 \\
\hline 32 & $0_{(100)}$ & $3_{(45)}$ & $2_{(75)}$ & $0_{(100)}$ & $0_{(100)}$ & $0_{(100)}$ & $0_{(100)}$ & 33.8 & $\mathrm{~S} 3_{(\mathrm{w}, \mathrm{s} 1)}$ \\
\hline 38 & $2_{(85)}$ & $0_{(100)}$ & $2_{(75)}$ & $0_{(100)}$ & $2_{(80)}$ & $0_{(100)}$ & $0_{(100)}$ & 50.0 & $\mathrm{~S} 3_{(\mathrm{t}, \mathrm{S} 1, \mathrm{~S} 3)}$ \\
\hline 40 & $0_{(100)}$ & $1_{(95)}$ & $2_{(65)}$ & $0_{(100)}$ & $0_{(100)}$ & $0_{(100)}$ & $0_{(100)}$ & 61.8 & $\mathrm{~S} 2_{(\mathrm{s} 1)}$ \\
\hline 50 & $2_{(85)}$ & $3_{(45)}$ & $2_{(70)}$ & $0_{(100)}$ & $0_{(100)}$ & $0_{(100)}$ & $0_{(100)}$ & 26.8 & $\mathrm{~S} 3_{(\mathrm{s} 1, \mathrm{w}, \mathrm{t})}$ \\
\hline 53 & $0_{(100)}$ & $0_{(100)}$ & $1_{(86)}$ & $0_{(100)}$ & $0_{(100)}$ & $0_{(100)}$ & $0_{(100)}$ & 86.0 & S1 \\
\hline 75 & $1_{(90)}$ & $0_{(100)}$ & $3_{(55)}$ & $0_{(100)}$ & $0_{(100)}$ & $0_{(100)}$ & $0_{(100)}$ & 49.5 & $\mathrm{~S} 3_{(\mathrm{s} 1, \mathrm{t})}$ \\
\hline 83 & $0_{(100)}$ & $0_{(100)}$ & $1_{(95)}$ & $2_{(75)}$ & $0_{(100)}$ & $0_{(100)}$ & $0_{(100)}$ & 71.3 & $\mathrm{~S} 2_{(\mathrm{s} 2)}$ \\
\hline 117 & $1_{(90)}$ & $0_{(100)}$ & $1_{(87)}$ & $0_{(100)}$ & $0_{(100)}$ & $0_{(100)}$ & $2_{(75)}$ & 58.7 & $\mathrm{~S} 2_{(\mathrm{n})}$ \\
\hline 131 & $0_{(100)}$ & $3_{(45)}$ & $1_{(90)}$ & $0_{(100)}$ & $0_{(100)}$ & $0_{(100)}$ & $0_{(100)}$ & 40.5 & $S 3_{(w)}$ \\
\hline \multicolumn{10}{|c|}{ Pediplain with deep soil } \\
\hline 4 & $0_{(100)}$ & $1_{(87)}$ & $2_{(75)}$ & $0_{(100)}$ & $0_{(100)}$ & $0_{(100)}$ & $0_{(100)}$ & 65.3 & $\mathrm{~S} 2_{(\mathrm{s} 1)}$ \\
\hline 5 & $0_{(100)}$ & $1_{(87)}$ & $1_{(90)}$ & $0_{(100)}$ & $0_{(100)}$ & $0_{(100)}$ & $2_{(85)}$ & 66.6 & $\mathrm{~S} 2_{(\mathrm{n})}$ \\
\hline 6 & $0_{(100)}$ & $2_{(80)}$ & $2_{(78)}$ & $0_{(100)}$ & $0_{(100)}$ & $0_{(100)}$ & $0_{(100)}$ & 62.4 & $\mathrm{~S} 2_{(\mathrm{s} 1, \mathrm{w})}$ \\
\hline 10 & $2(85)$ & $0_{(100)}$ & $3_{(48)}$ & $0_{(100)}$ & $0_{(100)}$ & $0_{(100)}$ & $0_{(100)}$ & 40.8 & $\mathrm{~S} 3_{(\mathrm{s} 1, \mathrm{t})}$ \\
\hline 14 & $2_{(85)}$ & $0_{(100)}$ & $2_{(73)}$ & $0_{(100)}$ & $0_{(100)}$ & $0_{(100)}$ & $3_{(58)}$ & 36 & $\mathrm{~S} 3_{(\mathrm{n}, \mathrm{s} 1, \mathrm{t})}$ \\
\hline 20 & $2(85)$ & $3_{(45)}$ & $2_{(60)}$ & $0_{(100)}$ & $0_{(100)}$ & $0_{(100)}$ & $0_{(100)}$ & 28 & $\mathrm{~S} 3_{(\mathrm{w}, \mathrm{s} 1, \mathrm{t})}$ \\
\hline 22 & $2(85)$ & $1_{(92)}$ & $2_{(65)}$ & $0_{(100)}$ & $0_{(100)}$ & $0_{(100)}$ & $0_{(100)}$ & 50.3 & $\mathrm{~S} 2_{(\mathrm{s} 1, \mathrm{t})}$ \\
\hline 43 & $0_{(100)}$ & $3_{(45)}$ & $2_{(75)}$ & $0_{(100)}$ & $0_{(100)}$ & $0_{(100)}$ & $0_{(100)}$ & 33.8 & $\mathrm{~S} 3_{(\mathrm{s} 1, \mathrm{w})}$ \\
\hline 171 & $0_{(100)}$ & $1_{(90)}$ & $2_{(65)}$ & $0_{(100)}$ & $0_{(100)}$ & $0_{(100)}$ & $0_{(100)}$ & 58.5 & $\mathrm{~S} 2_{(\mathrm{s} 1, \mathrm{t})}$ \\
\hline \multicolumn{10}{|c|}{ Pediplain with shallow soil } \\
\hline 103 & $0_{(100)}$ & $3_{(45)}$ & $1_{(88)}$ & $0_{(100)}$ & $0_{(100)}$ & $0_{(100)}$ & $0_{(100)}$ & 39.6 & $S 3_{(w)}$ \\
\hline 143 & $0_{(100)}$ & $0_{(100)}$ & $2_{(83)}$ & $0_{(100)}$ & $0_{(100)}$ & $0_{(100)}$ & $0_{(100)}$ & 83.0 & $\mathrm{~S} 1$ \\
\hline 123 & $2(85)$ & $1_{(90)}$ & $2(84)$ & $0_{(100)}$ & $1_{(90)}$ & $0_{(100)}$ & $1_{(90)}$ & 50.1 & $\mathrm{~S} 2_{(\mathrm{t}, \mathrm{s} 1)}$ \\
\hline 128 & $0_{(100)}$ & $2_{(70)}$ & $2(63)$ & $1_{(95)}$ & $0_{(100)}$ & $0_{(100)}$ & $0_{(100)}$ & 41.9 & $\mathrm{~S} 3_{(\mathrm{s} 1, \mathrm{w})}$ \\
\hline 184 & $0_{(100)}$ & $2_{(65)}$ & $2_{(75)}$ & $2_{(75)}$ & $0_{(100)}$ & $0_{(100)}$ & $2_{(80)}$ & 29.3 & $\mathrm{~S} 3_{(\mathrm{w}, \mathrm{s} 2, \mathrm{n})}$ \\
\hline
\end{tabular}




\begin{tabular}{|c|c|c|c|c|c|c|c|c|c|}
\hline 192 & $1_{(90)}$ & $3_{(52)}$ & $0_{(100)}$ & $2_{(75)}$ & $0_{(100)}$ & $0_{(100)}$ & $2_{(80)}$ & 28.1 & $\mathrm{~S} 3_{(\mathrm{w}, \mathrm{s} 2)}$ \\
\hline \multicolumn{10}{|c|}{ Active slope } \\
\hline 70 & $0_{(100)}$ & $0_{(100)}$ & $2_{(79)}$ & $0_{(100)}$ & $0_{(100)}$ & $0_{(100)}$ & $0_{(98)}$ & 73.5 & $S 2_{(s 1)}$ \\
\hline 72 & $0_{(100)}$ & $0_{(100)}$ & $1_{(85)}$ & $1_{(90)}$ & $0_{(100)}$ & $0_{(100)}$ & $2_{(75)}$ & 57.4 & $\mathrm{~S} 2_{(\mathrm{s} 1, \mathrm{n})}$ \\
\hline \multicolumn{10}{|c|}{ Hills and rock outcrops } \\
\hline 57 & $0_{(100)}$ & $0_{(100)}$ & $2_{(83)}$ & $0_{(100)}$ & $0_{(100)}$ & $0_{(100)}$ & $0_{(100)}$ & 83 & S1 \\
\hline 94 & $2_{(85)}$ & $2_{(85)}$ & $2_{(79)}$ & $1_{(95)}$ & $0_{(100)}$ & $0_{(100)}$ & $1_{(90)}$ & 48.8 & $\mathrm{~S} 3_{(\mathrm{t}, \mathrm{w}, \mathrm{s} 1)}$ \\
\hline 114 & $1_{(90)}$ & $0_{(100)}$ & $2_{(75)}$ & $0_{(100)}$ & $0_{(100)}$ & $0_{(100)}$ & $1_{(90)}$ & 60.8 & $\mathrm{~S} 2_{(\mathrm{s} 1)}$ \\
\hline 221 & $3_{(50)}$ & $0_{(100)}$ & $1_{(90)}$ & $0_{(100)}$ & $1_{(90)}$ & $0_{(100)}$ & $0_{(100)}$ & 40.5 & $\mathrm{~S} 3_{(\mathrm{t})}$ \\
\hline 224 & $3_{(50)}$ & $0_{(100)}$ & $2_{(75)}$ & $0_{(100)}$ & $0_{(100)}$ & $0_{(100)}$ & $0_{(100)}$ & 37.5 & $\mathrm{~S} 3_{(\mathrm{t}, \mathrm{s} 1)}$ \\
\hline \multicolumn{10}{|c|}{ Footslope } \\
\hline 36 & $22_{(80)}$ & $2_{(80)}$ & $2_{(77)}$ & $0_{(100)}$ & $0_{(100)}$ & $0_{(100)}$ & $0_{(100)}$ & 46.2 & $\mathrm{~S} 3_{(\mathrm{t}, \mathrm{s} 1, \mathrm{w})}$ \\
\hline 37 & $3_{(60)}$ & $2_{(80)}$ & $1_{(90)}$ & $0_{(100)}$ & $0_{(100)}$ & $0_{(100)}$ & $0_{(100)}$ & 43.2 & $\mathrm{~S} 3_{(\mathrm{t}, \mathrm{w})}$ \\
\hline 56 & $2_{(70)}$ & $0_{(100)}$ & $2_{(81)}$ & $0_{(100)}$ & $0_{(100)}$ & $0_{(100)}$ & $1_{(90)}$ & 50.0 & $\mathrm{~S} 3_{(\mathrm{t}, \mathrm{s} 1, \mathrm{n})}$ \\
\hline 109 & $1_{(90)}$ & $0_{(100)}$ & $1_{(89)}$ & $2_{(75)}$ & $0_{(100)}$ & $0_{(100)}$ & $0_{(100)}$ & 60.1 & $\mathrm{~S} 2_{(\mathrm{s} 2)}$ \\
\hline 124 & $1_{(90)}$ & $0_{(100)}$ & $1_{(94)}$ & $0_{(100)}$ & $0_{(100)}$ & $0_{(100)}$ & $0_{(100)}$ & 84.6 & S1 \\
\hline 141 & $1_{(85)}$ & $2_{(75)}$ & $2_{(69)}$ & $2_{(75)}$ & $0_{(100)}$ & $0_{(100)}$ & $1_{(90)}$ & 29.7 & $\mathrm{~S} 3_{(\mathrm{s} 1, \mathrm{~s} 2, \mathrm{w}, \mathrm{t})}$ \\
\hline 146 & $1_{(90)}$ & $0_{(100)}$ & $2_{(77)}$ & $2_{(75)}$ & $0_{(100)}$ & $0_{(100)}$ & $0_{(100)}$ & 50.8 & $\mathrm{~S} 3_{(\mathrm{s} 1, \mathrm{~s} 2)}$ \\
\hline 147 & $0_{(100)}$ & $1_{(90)}$ & $2_{(82)}$ & $1_{(95)}$ & $0_{(100)}$ & $0_{(100)}$ & $1_{(90)}$ & 63.1 & $\mathrm{~S} 2_{(\mathrm{s} 1)}$ \\
\hline 155 & $1_{(90)}$ & $2_{(75)}$ & $2_{(79)}$ & $2_{(75)}$ & $0_{(100)}$ & $0_{(100)}$ & $2_{(75)}$ & 30 & $\mathrm{~S} 3_{(\mathrm{w}, \mathrm{s} 1, \mathrm{~s} 2, \mathrm{n})}$ \\
\hline 156 & $2_{(85)}$ & $0_{(100)}$ & $1_{(90)}$ & $1_{(95)}$ & $0_{(100)}$ & $0_{(100)}$ & $0_{(100)}$ & 72.7 & $\mathrm{~S} 2_{(\mathrm{t})}$ \\
\hline 169 & $2_{(85)}$ & $2_{(68)}$ & $2_{(68)}$ & $3_{(55)}$ & $1_{(90)}$ & $0_{(100)}$ & $1_{(90)}$ & 17.5 & $\mathrm{~N} 1_{(\mathrm{w}, \mathrm{s} 1, \mathrm{t})}$ \\
\hline 173 & $1_{(90)}$ & $2_{(68)}$ & $1_{(87)}$ & $0_{(100)}$ & $0_{(100)}$ & $0_{(100)}$ & $0_{(100)}$ & 53.3 & $\mathrm{~S} 2_{(\mathrm{w})}$ \\
\hline 174 & $0_{(100)}$ & $1_{(90)}$ & $1_{(95)}$ & $0_{(100)}$ & $0_{(100)}$ & $0_{(100)}$ & $2_{(85)}$ & 72.7 & $\mathrm{~S} 2_{(\mathrm{n})}$ \\
\hline 183 & $1_{(90)}$ & $3_{(45)}$ & $2_{(75)}$ & $0_{(100)}$ & $0_{(100)}$ & $0_{(100)}$ & $1_{(90)}$ & 27.3 & $\mathrm{~S} 3_{(\mathrm{w}, \mathrm{s} 1)}$ \\
\hline 186 & $0_{(100)}$ & $2(85)$ & $2_{(83)}$ & $2_{(85)}$ & $0_{(100)}$ & $0_{(100)}$ & $2_{(80)}$ & 54 & $\mathrm{~S} 2_{(\mathrm{w}, \mathrm{s} 1, \mathrm{~s} 2)}$ \\
\hline 193 & $3_{(50)}$ & $1_{(90)}$ & $1_{(90)}$ & $2_{(75)}$ & $0_{(100)}$ & $0_{(100)}$ & $3_{(85)}$ & 15.9 & $\mathrm{~N} 1_{(\mathrm{t}, \mathrm{s} 2, \mathrm{n})}$ \\
\hline 198 & $3_{(50)}$ & $3_{(45)}$ & $2_{(68)}$ & $3_{(55)}$ & $0_{(100)}$ & $0_{(100)}$ & $0_{(100)}$ & 7.57 & $\mathrm{~N} 1_{(\mathrm{t}, \mathrm{s} 2)}$ \\
\hline 205 & $0_{(100)}$ & $2_{(75)}$ & $2_{(82)}$ & $3_{(55)}$ & $1_{(90)}$ & $0_{(100)}$ & $2_{(60)}$ & 16.2 & $\mathrm{~N} 1_{(\mathrm{s} 2 \mathrm{n}, \mathrm{w}, \mathrm{S} 3)}$ \\
\hline 211 & $0_{(100)}$ & $0_{(98)}$ & $2_{(83)}$ & $0_{(100)}$ & $1_{(90)}$ & $0_{(100)}$ & $0_{(100)}$ & 73 & $\mathrm{~S} 2_{(\mathrm{s} 1)}$ \\
\hline 220 & $3_{(50)}$ & $2_{(75)}$ & $1_{(90)}$ & $0_{(100)}$ & $0_{(100)}$ & $0_{(100)}$ & $0_{(100)}$ & 33.8 & $\mathrm{~S} 3_{(\mathrm{t}, \mathrm{w})}$ \\
\hline 223 & $3_{(50)}$ & $2_{(75)}$ & $2_{(81)}$ & $4_{(30)}$ & $0_{(100)}$ & $0_{(100)}$ & $0_{(100)}$ & 9.11 & $\mathrm{~N} 1_{(\mathrm{t}, \mathrm{s} 2)}$ \\
\hline \multicolumn{10}{|c|}{ Terraced } \\
\hline 71 & $0_{(100)}$ & $0_{(100)}$ & $2_{(68)}$ & $0_{(100)}$ & $0_{(100)}$ & $0_{(100)}$ & $0_{(100)}$ & 68 & $\mathrm{~S} 2_{(\mathrm{s} 1)}$ \\
\hline 86 & $2_{(85)}$ & $0_{(100)}$ & $1_{(90)}$ & $1_{(95)}$ & $0_{(100)}$ & $0_{(100)}$ & $0_{(100)}$ & 72.7 & $\mathrm{~S} 2_{(\mathrm{t})}$ \\
\hline 105 & $1_{(90)}$ & $2(75)$ & $1_{(91)}$ & $1_{(95)}$ & $0_{(100)}$ & $0_{(100)}$ & $0_{(100)}$ & 58.4 & $\mathrm{~S} 2_{\mathrm{w}}$ \\
\hline 106 & $2_{(85)}$ & $0_{(98)}$ & $2_{(75)}$ & $1_{(95)}$ & $0_{(100)}$ & $0_{(100)}$ & $0_{(100)}$ & 59.4 & $\mathrm{~S} 2_{(\mathrm{s} 1, \mathrm{t})}$ \\
\hline 110 & $1_{(90)}$ & $0_{(100)}$ & $1_{(85)}$ & $0_{(100)}$ & $0_{(100)}$ & $0_{(100)}$ & $0_{(100)}$ & 76.7 & S1 \\
\hline 118 & $2_{(85)}$ & $0_{(100)}$ & $1_{(90)}$ & $1_{(95)}$ & $0_{(100)}$ & $0_{(100)}$ & $3_{(58)}$ & 42.2 & $\mathrm{~S} 3_{(\mathrm{n}, \mathrm{t})}$ \\
\hline 129 & $2_{(85)}$ & $0_{(100)}$ & $3_{(59)}$ & $1_{(95)}$ & $1_{(95)}$ & $0_{(100)}$ & $0_{(100)}$ & 45.2 & $\mathrm{~S} 3_{(\mathrm{s} 1, \mathrm{t})}$ \\
\hline 136 & $1_{(90)}$ & $1_{(87)}$ & $2_{(82)}$ & $0_{(100)}$ & $0_{(100)}$ & $0_{(100)}$ & $0_{(100)}$ & 64.2 & $\mathrm{~S} 2_{(\mathrm{s} 1)}$ \\
\hline 148 & $1_{(90)}$ & $2_{(80)}$ & $1_{(94)}$ & $0_{(100)}$ & $0_{(100)}$ & $0_{(100)}$ & $0_{(100)}$ & 67.7 & $\mathrm{~S} 2_{(\mathrm{w})}$ \\
\hline 164 & $3_{(50)}$ & $2_{(80)}$ & $2_{(84)}$ & $0_{(100)}$ & $0_{(100)}$ & $0_{(100)}$ & $0_{(100)}$ & 33.5 & $\mathrm{~S} 3_{(\mathrm{t}, \mathrm{w}, \mathrm{s} 1)}$ \\
\hline 175 & $3_{(50)}$ & $2_{(75)}$ & $0_{(100)}$ & $0_{(100)}$ & $0_{(100)}$ & $0_{(100)}$ & $0_{(100)}$ & 37.5 & $\mathrm{~S} 3_{(\mathrm{t}, \mathrm{w})}$ \\
\hline \multicolumn{10}{|c|}{ Lava field and volcanic hills } \\
\hline 225 & $3_{(50)}$ & $0_{(100)}$ & $1_{(95)}$ & $0_{(100)}$ & $0_{(100)}$ & $0_{(100)}$ & $1_{(90)}$ & 42.8 & $\mathrm{~S} 3_{(\mathrm{t})}$ \\
\hline 226 & $3_{(50)}$ & $0_{(100)}$ & $1_{(95)}$ & $0_{(100)}$ & $0_{(100)}$ & $0_{(100)}$ & $0_{(100)}$ & 47.5 & $\mathrm{~S} 3_{(\mathrm{t})}$ \\
\hline \multicolumn{10}{|c|}{ Alluvial fan } \\
\hline 190 & $0_{(100)}$ & $0_{(100)}$ & $2_{(74)}$ & $0_{(100)}$ & $0_{(100)}$ & $0_{(100)}$ & $0_{(100)}$ & 74 & $\mathrm{~S} 2_{(\mathrm{s} 1, \mathrm{n})}$ \\
\hline 197 & $0_{(100)}$ & $2(80)$ & $2_{(75)}$ & $2_{(75)}$ & $0_{(100)}$ & $0_{(100)}$ & $2_{(80)}$ & 48 & $\mathrm{~S} 3_{(\mathrm{s} 1, \mathrm{~s} 2)}$ \\
\hline 204 & $0_{(100)}$ & $2(83)$ & $1_{(90)}$ & $0_{(100)}$ & $2_{(80)}$ & $0_{(100)}$ & $2_{(80)}$ & 47.8 & $\mathrm{~S} 33_{(\mathrm{w}, \mathrm{s} 3, \mathrm{n})}$ \\
\hline 208 & $0_{(100)}$ & $0_{(100)}$ & $2_{(61)}$ & $0_{(100)}$ & $0_{(100)}$ & $0_{(100)}$ & $0_{(100)}$ & 61 & $\mathrm{~S} 2_{(\mathrm{s} 1)}$ \\
\hline 218 & $0_{(100)}$ & $1_{(95)}$ & $2_{(88)}$ & $0_{(100)}$ & $0_{(100)}$ & $0_{(100)}$ & $0_{(100)}$ & 83.6 & S1 \\
\hline
\end{tabular}

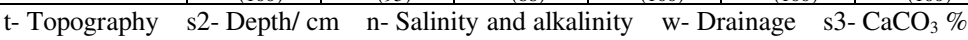

Ci- Capability Index s1- Texture s4-Coarse fragments \% 
slope $100 \%$, hills and rock outcrops $18 \%$, footslopes $26 \%$, terraced $63 \%$ and, alluvial fan $36 \%$ ). Marginally suitable land (S3), lands within this capability class have limitations which in aggregate are severe (sabkhat $39.1 \%$, coastal plain $69 \%$, alluvial plain $44 \%$, wadis $27 \%$, pediplain with deep soil $49 \%$, pediplain with shallow soil $44 \%$, hills and rock outcrops $64 \%$, footslopes $32 \%$, Lava field and volcanic hills $100 \%$, terraced $26 \%$ and alluvial fan $46 \%$ ). Currently not suitable (N1), lands within this class have severe limitations and can not be corrected at currently acceptable cost, these soils require high input levels for leaching salts and improving drainability. After good soil management these soils can be cultivated with highly salt tolerant crops (sabkhat 60.9\%, coastal plain 20\%, wadis $11 \%$ and footslopes 23\%). The permentally unsuitable (N2) (wadis 11\%).

\subsection{The qualitative evaluation system for irrigation according to FAO (1979 and 1983).}

In this classification, land classes for irrigation farming are related to the U. S. Bureau of Reclamation (USBR) classes (1951), Table (4).

The system uses six classes collected in three class levels as follows:

- S1, S2 and S3 are considered suitable for any type of irrigation systems (i. e. surface, drip and sprinkler), they are particularly suitable for surface irrigation.

- Class Sc is suitable for non- traditional types of irrigation systems (i.e. drip and sprinkler). suitable soils represent less area $16.2 \%$ for currently not suitable and $2.2 \%$ permanently not suitable of the total area.

The results of soil suitability evaluation showed 6 classes. The high suitable soils represent $13 \%$ of total area, while most of the area includes suitable soils with restriction (36\% of total area). The non-suitable soils represent less area $(2.2 \%)$.

In the study area, the results of the two systems are collected in the following land suitability classes:

- Highly suitable land (S1)

Land within this class has no significant limitation to sustained application of a given use or only minor limitations that will not significantly reduce productivity or benefits, and will not raise inputs to unacceptable level. Land units may have $3 / 4$ slight limitations and land indice is higher than 75 . In the studied area, land within this class is quite limited, these soils have the requirements of highly suitable land $(\mathrm{S} 1), i$. e. soil is flat, very deep, well drained, with optimal infiltration rate $(1.12 \mathrm{~cm} / \mathrm{h})$, silty clay loam textured, rich in organic matter (2.5\%), and free of salts (Table 6). The capability index is 96, (Table 3). Gravity irrigation could be easily applied in these soils (S1) (Table 5).

- Moderately suitable land (S2)

Soils within this suitability class have moderate limitations for sustained application of a given use, the limitations will reduce productivity or benefits and increase required inputs to the extent

Table (4): Correction between USBR and FAO land classification.

\begin{tabular}{|r|c|c|}
\hline FAO & Class definition & USBR \\
\hline S1 & Highly suitable & Class 1 \\
\hline S2 & Moderately suitable & Class 2 \\
\hline S3 & Marginally suitable & Class 3 \\
\hline Sc & Special use & Class 4 \\
\hline N1 & Not suitable at present but potentially suitable & Class 5 \\
\hline N2 & Actiually and potentially unsuitable & Class 6 \\
\hline
\end{tabular}

- N1 and N2 are unsuitable land for irrigated agriculture.

Table (5) shows the results of the land suitability for irrigation according to FAO (1979 and 1983). While Table (6) shows that the high suitable soils represent $13 \%$, the moderately suitable soils represent $16.8 \%$, the marginally suitable soils represent $15.8 \%$ of the total area, while most of the area includes suitable soils with restriction (36\% of total area). The non- that the overall advantage to be gained from the use will be appreciably inferior to expect on S1 class. Moderately suitable; land may have more than four slight limitations and/or two or three moderate limitations. The land indice mostly situated between 50 and 75 . Most of these soils are characterized by flat surface, very deep, loam to clay loam textured, well drained, with optimum or nearly optimum infiltration rates $(0.8-7.0 \mathrm{~cm} / \mathrm{h})$ and free of salts. Agriculture limitations are 
Table (5): Land Suitability for Irrigated Agriculture According to FAO (1979 and 1983).

\begin{tabular}{|c|c|c|c|c|c|c|c|c|c|c|c|}
\hline Profil No. & $\mathrm{t}$ & $\mathrm{w}$ & s1 & s1 & $\mathrm{s} 2$ & s2! & S3 & s4 & s4 & $\mathrm{n}$ & Subclass \\
\hline \multicolumn{12}{|c|}{ Sabkhat } \\
\hline 1 & S1 & N1 & $\mathrm{S} 1$ & S1 & S1 & S3 & S1 & S1 & S1 & N1 & $\mathrm{N} 1(\mathrm{w}, \mathrm{n}, \mathrm{s} 2)$ \\
\hline 47 & $\mathrm{~S} 1$ & S3 & $\mathrm{S} 1$ & S2 & S2 & & S1 & S1 & S1 & N1 & N1(n) \\
\hline 48 & S1 & N1 & S1 & S2 & S3 & & S1 & S1 & S1 & N1 & $\mathrm{N} 1(\mathrm{n}, \mathrm{s} 2)$ \\
\hline 63 & S3 & N1 & $\mathrm{SC}$ & S3 & S2 & & S1 & S1 & S1 & S1 & $\mathrm{SC}(\mathrm{s} 1, \mathrm{t})$ \\
\hline 166 & S1 & N1 & S2 & S1 & S3 & & S2 & S1 & S1 & SC & $\mathrm{N} 1(\mathrm{w}, \mathrm{n}, \mathrm{s} 1)$ \\
\hline 194 & $\mathrm{~S} 1$ & N1 & SC & $\mathrm{SC}$ & S2 & & S2 & S1 & S1 & N1 & $\mathrm{N} 1(\mathrm{n}, \mathrm{w}, \mathrm{s} 1)$ \\
\hline \multicolumn{12}{|c|}{ Coastal plain } \\
\hline 2 & S3 & S2 & $\mathrm{SC}$ & $\mathrm{SC}$ & $\mathrm{S} 1$ & S1 & S1 & S1 & S1 & S1 & $\mathrm{SC}(\mathrm{s} 1, \mathrm{t})$ \\
\hline 17 & $\mathrm{SC}$ & S1 & S2 & S1 & S1 & S1 & S1 & S1 & S1 & S2 & $\mathrm{SC}(\mathrm{t})$ \\
\hline 24 & S1 & S1 & S1 & S1 & S1 & S1 & S1 & S1 & S1 & SC & SC(n) \\
\hline 46 & S3 & $\mathrm{SC}$ & $\mathrm{S} 2$ & S1 & & S1 & S1 & S1 & S1 & $\mathrm{SC}$ & $\mathrm{SC}(\mathrm{t}, \mathrm{w}, \mathrm{n})$ \\
\hline 49 & S3 & $\mathrm{SC}$ & $\mathrm{S} 1$ & S1 & & S1 & S1 & S1 & S1 & S1 & $\mathrm{SC}(\mathrm{t}, \mathrm{w})$ \\
\hline 180 & S1 & $\mathrm{SC}$ & $\mathrm{S} 1$ & S1 & & S1 & S2 & S1 & S1 & N1 & $\mathrm{N} 1(\mathrm{n}, \mathrm{w})$ \\
\hline 188 & S1 & S1 & S1 & S2 & & S1 & S1 & S1 & S1 & S1 & S2(s1) \\
\hline 216 & S1 & S2 & S1 & S1 & & S1 & S2 & S1 & S1 & S1 & $\mathrm{S} 2(\mathrm{w}, \mathrm{s} 3)$ \\
\hline \multicolumn{12}{|c|}{ Wadis } \\
\hline 3 & $\mathrm{~S} 1$ & S2 & $\mathrm{SC}$ & S1 & S1 & S1 & S1 & S1 & S1 & S1 & $\mathrm{SC}(\mathrm{s} 1)$ \\
\hline 25 & $\mathrm{~S} 1$ & S1 & $\mathrm{S} 1$ & S1 & $\mathrm{S} 1$ & S1 & S1 & S1 & S1 & S1 & S1 \\
\hline 30 & S1 & S1 & S1 & $\mathrm{SC}$ & S1 & S1 & S1 & S1 & S1 & S1 & $\mathrm{SC}(\mathrm{s} 1)$ \\
\hline 133 & S1 & $\mathrm{S} 1$ & S1 & $\mathrm{S} 1$ & & S1 & S1 & S1 & S1 & S1 & S1 \\
\hline 134 & $\mathrm{~S} 1$ & S1 & $\mathrm{S} 1$ & S1 & & S3 & S1 & $\mathrm{N} 2$ & $\mathrm{~N} 2$ & S2 & $\mathrm{N} 2(\mathrm{~s} 4, \mathrm{~s} 2)$ \\
\hline 162 & S1 & $\mathrm{SC}$ & S3 & S2 & & S1 & S1 & $\mathrm{SC}$ & $\mathrm{SC}$ & S1 & SC(w,s5) \\
\hline 191 & $\mathrm{~S} 1$ & S1 & $\mathrm{S} 1$ & S1 & & $\mathrm{SC}$ & S1 & S1 & S1 & S1 & $\mathrm{SC}(\mathrm{s} 2)$ \\
\hline 199 & N1 & S1 & SC & S1 & & SC & S1 & S1 & S1 & S1 & $\mathrm{N} 1(\mathrm{t}, \mathrm{s} 5, \mathrm{~s} 2)$ \\
\hline 209 & $\mathrm{~S} 1$ & S1 & $\mathrm{S} 1$ & $\mathrm{SC}$ & & N1 & S1 & S1 & S1 & S1 & $\mathrm{N} 1(\mathrm{~s} 2, \mathrm{~s} 1)$ \\
\hline 219 & $\mathrm{~S} 1$ & S1 & SC & $\mathrm{SC}$ & & $\mathrm{N} 2$ & S2 & S1 & S1 & S1 & $\mathrm{N} 2(\mathrm{~s} 2)$ \\
\hline \multicolumn{12}{|c|}{ Alluvial plain } \\
\hline 26 & S1 & S2 & $\mathrm{S} 1$ & S1 & S1 & S1 & S1 & S1 & S1 & S1 & S2(w) \\
\hline 27 & S1 & S2 & $\mathrm{S} 1$ & S1 & S1 & S1 & S1 & S1 & S1 & S1 & S2(w) \\
\hline 28 & $\mathrm{~S} 1$ & S1 & SC & S1 & S1 & S1 & S1 & S1 & S1 & S1 & $\mathrm{SC}(\mathrm{s} 1)$ \\
\hline 29 & S1 & S2 & S1 & S1 & S1 & S1 & S1 & S1 & S1 & S1 & S2(w) \\
\hline 31 & $\mathrm{~S} 1$ & S1 & $\mathrm{S} 1$ & S1 & S1 & S1 & S1 & S1 & S1 & S1 & S1 \\
\hline 32 & S1 & $\mathrm{SC}$ & S1 & S1 & S1 & S1 & S1 & S1 & S1 & S1 & $\mathrm{SC}(\mathrm{w})$ \\
\hline 38 & S3 & S1 & S1 & S1 & S1 & S1 & SC & S1 & S1 & S1 & $\mathrm{SC}(\mathrm{t}, \mathrm{s} 3)$ \\
\hline 40 & $\mathrm{~S} 1$ & S1 & $\mathrm{S} 1$ & S2 & S1 & S1 & S1 & S1 & S1 & S1 & S2(s1) \\
\hline 50 & SC & $\mathrm{SC}$ & S2 & S2 & & S1 & S1 & S1 & S1 & S1 & $\mathrm{SC}(\mathrm{t}, \mathrm{w})$ \\
\hline 53 & $\mathrm{~S} 1$ & $\mathrm{~S} 1$ & $\mathrm{~S} 1$ & S1 & & S1 & S1 & S1 & S1 & S1 & S1 \\
\hline 75 & S3 & $\mathrm{SC}$ & S1 & $\mathrm{SC}$ & & S1 & S1 & S1 & S1 & S1 & $\mathrm{SC}(\mathrm{w}, \mathrm{s}, \mathrm{t})$ \\
\hline 83 & S1 & $\mathrm{S} 1$ & S1 & S1 & & S2 & S1 & S1 & S1 & S1 & S2(s2) \\
\hline 117 & S3 & S1 & $\mathrm{S} 1$ & S1 & & S1 & S1 & $\mathrm{S} 2$ & S1 & S3 & $\mathrm{S} 3(\mathrm{n}, \mathrm{t})$ \\
\hline 131 & S1 & S3 & S1 & S2 & & S1 & S1 & S1 & S1 & S1 & $\mathrm{S} 3(\mathrm{w}, \mathrm{s} 1)$ \\
\hline \multicolumn{12}{|c|}{ Pediplain with deep soil } \\
\hline 4 & $\mathrm{~S} 1$ & S1 & $\mathrm{S} 1$ & S1 & S1 & S1 & S1 & S1 & S1 & S1 & S1 \\
\hline 5 & $\mathrm{~S} 1$ & S1 & $\mathrm{S} 1$ & S1 & S1 & S1 & S1 & S1 & S1 & S3 & S3(n) \\
\hline 6 & S1 & S1 & S1 & S1 & S1 & S1 & S1 & S1 & S2 & S1 & S2(s4) \\
\hline 10 & S3 & S1 & SC & $\mathrm{SC}$ & S1 & S1 & S1 & S1 & S1 & S1 & $\mathrm{SC}(\mathrm{s} 1, \mathrm{t})$ \\
\hline 14 & S3 & S1 & $\mathrm{S} 2$ & S1 & S1 & S1 & S1 & S1 & S1 & $\mathrm{SC}$ & $\mathrm{SC}(\mathrm{n}, \mathrm{t})$ \\
\hline 20 & S3 & $\mathrm{SC}$ & S3 & S1 & S1 & S1 & S1 & S1 & S1 & S1 & $\mathrm{SC}(\mathrm{s} 1, \mathrm{w}, \mathrm{t})$ \\
\hline 22 & S3 & $\mathrm{S} 1$ & S1 & S3 & S1 & S1 & S1 & S1 & S1 & S1 & $\mathrm{S} 3(\mathrm{~s} 1, \mathrm{t})$ \\
\hline 43 & S1 & $\mathrm{SC}$ & S1 & S2 & & S1 & S1 & S1 & S1 & S1 & $\mathrm{SC}(\mathrm{w})$ \\
\hline 171 & $\mathrm{~S} 1$ & S1 & $\mathrm{S} 2$ & S2 & & S1 & S1 & S1 & S1 & S1 & $\mathrm{S} 2(\mathrm{~s} 1, \mathrm{~s} 5)$ \\
\hline \multicolumn{12}{|c|}{ Pediplain with shallow soil } \\
\hline 103 & $\mathrm{~S} 1$ & S3 & $\mathrm{S} 1$ & S1 & & S1 & S1 & S1 & S1 & S1 & S3(w) \\
\hline 123 & $\mathrm{SC}$ & S1 & $\mathrm{S} 1$ & S1 & & S1 & SC & S2 & S1 & S3 & $\mathrm{SC}(\mathrm{t}, \mathrm{s} 3, \mathrm{~s} 5, \mathrm{~N})$ \\
\hline 128 & $\mathrm{~S} 1$ & S2 & $\mathrm{S} 2$ & S2 & & S1 & S1 & S1 & S1 & S1 & $\mathrm{S} 2(\mathrm{~s} 1, \mathrm{w})$ \\
\hline 143 & S1 & S1 & $\mathrm{S} 1$ & S1 & & S1 & S1 & $\mathrm{S} 2$ & S2 & S1 & $\mathrm{S} 2(\mathrm{~s} 4)$ \\
\hline
\end{tabular}




\begin{tabular}{|c|c|c|c|c|c|c|c|c|c|c|c|}
\hline 184 & S1 & S2 & S1 & S1 & & S1 & S1 & $\mathrm{SC}$ & $\mathrm{SC}$ & S1 & $\mathrm{SC}(\mathrm{s} 2, \mathrm{w}, \mathrm{s} 5)$ \\
\hline 192 & S3 & S3 & S1 & S1 & & SC & S1 & $\mathrm{SC}$ & S1 & $\mathrm{SC}$ & $\mathrm{SC}(\mathrm{t}, \mathrm{w}, \mathrm{s} 2, \mathrm{~s} 5, \mathrm{n})$ \\
\hline \multicolumn{12}{|c|}{ Active slope } \\
\hline 70 & S1 & S1 & S1 & $\mathrm{S} 2$ & & S1 & S1 & S1 & S1 & S2 & $\mathrm{S} 2(\mathrm{n}, \mathrm{s} 1)$ \\
\hline 72 & S1 & S1 & S1 & S1 & & S2 & S1 & S1 & S1 & S3 & $\mathrm{S} 3(\mathrm{n}, \mathrm{s} 2)$ \\
\hline \multicolumn{12}{|c|}{ Hills and rock outcrops } \\
\hline 57 & S1 & S1 & S1 & S1 & & S1 & \begin{tabular}{|l|l} 
S1 \\
\end{tabular} & S1 & S1 & S1 & S1 \\
\hline 94 & SC & S2 & $\mathrm{SC}$ & S1 & & S1 & S1 & S1 & S1 & S2 & $\mathrm{SC}(\mathrm{t}, \mathrm{s} 1)$ \\
\hline 114 & S3 & S1 & S1 & S2 & & S2 & S1 & S2 & S1 & $\mathrm{SC}$ & $\mathrm{SC}(\mathrm{t}, \mathrm{s} 5, \mathrm{n})$ \\
\hline 221 & N1 & S1 & S1 & S1 & & S1 & SC & S1 & S1 & S1 & $\mathrm{N} 1(\mathrm{t}, \mathrm{s} 5, \mathrm{~s} 3)$ \\
\hline 224 & N1 & S1 & S1 & S1 & & S1 & S1 & S1 & S1 & S2 & $\mathrm{N} 1(\mathrm{t}, \mathrm{s} 4)$ \\
\hline \multicolumn{12}{|c|}{ Footslope } \\
\hline 36 & S3 & S1 & S2 & S1 & S1 & S1 & \begin{tabular}{|l|} 
S1 \\
\end{tabular} & $\mathrm{SC}$ & S1 & S1 & $\mathrm{SC}$ \\
\hline 37 & SC & S1 & S1 & S1 & S1 & S1 & S1 & S1 & S1 & S1 & $\mathrm{SC}(\mathrm{t})$ \\
\hline 56 & N1 & S1 & S1 & S2 & & S1 & S1 & S1 & S1 & S3 & $\mathrm{N} 1(\mathrm{t}, \mathrm{s} 5, \mathrm{n})$ \\
\hline 109 & S3 & S1 & S1 & S1 & & S2 & S1 & S1 & S3 & S1 & $\mathrm{SC}(\mathrm{t}, \mathrm{s} 5)$ \\
\hline 124 & S3 & S1 & S1 & S1 & & S1 & S1 & S1 & S1 & S1 & $\mathrm{SC}(\mathrm{t}, \mathrm{s} 5)$ \\
\hline 140 & S1 & S1 & S1 & S1 & & S1 & S1 & S1 & S1 & S1 & S1 \\
\hline 141 & SC & S2 & S1 & S2 & & S2 & S1 & S2 & $\mathrm{SC}$ & S3 & $\mathrm{SC}(\mathrm{t}, \mathrm{s} 5, \mathrm{n})$ \\
\hline 146 & S3 & S1 & S1 & S1 & & S2 & \begin{tabular}{|l|} 
S1 \\
\end{tabular} & SC & SC & S2 & $\mathrm{SC}(\mathrm{t}, \mathrm{s} 5)$ \\
\hline 147 & S1 & S1 & S1 & S1 & & S1 & S1 & S1 & N1 & S3 & $\mathrm{N} 1(\mathrm{~s} 4, \mathrm{n})$ \\
\hline 155 & S3 & S2 & S1 & S2 & & S3 & S1 & S1 & S1 & N1 & $\mathrm{N} 1(\mathrm{t}, \mathrm{n}, \mathrm{s} 2)$ \\
\hline 156 & SC & S1 & S1 & S1 & & S1 & S1 & S1 & S1 & S1 & $\mathrm{SC}(\mathrm{t}, \mathrm{s} 5)$ \\
\hline 169 & $\mathrm{SC}$ & S2 & S1 & S1 & & N1 & S2 & SC & S3 & S3 & $\mathrm{N} 1(\mathrm{t}, \mathrm{s} 5, \mathrm{~s} 2, \mathrm{n})$ \\
\hline 173 & S3 & S2 & S1 & S1 & & S1 & S1 & S1 & S1 & S1 & $\mathrm{S} 3(\mathrm{t}, \mathrm{w})$ \\
\hline 174 & S1 & S1 & S1 & S1 & & S1 & S1 & S1 & S1 & S3 & S3(n) \\
\hline 183 & S3 & S3 & S1 & S2 & & S1 & S1 & S1 & S1 & S3 & $\mathrm{N} 1(\mathrm{t}, \mathrm{w}, \mathrm{n}, \mathrm{s} 5)$ \\
\hline 186 & S1 & S2 & S1 & S2 & & SC & S1 & S1 & S1 & S3 & $\mathrm{SC}(\mathrm{s} 2, \mathrm{n})$ \\
\hline 193 & N1 & S1 & S1 & S1 & & SC & S2 & S1 & S1 & N1 & N1(t,s2,n) \\
\hline 198 & N1 & SC & S2 & S2 & & $\mathrm{SC}$ & $\mathrm{SC}$ & S1 & S1 & S1 & $\mathrm{N} 1(\mathrm{t}, \mathrm{w}, \mathrm{s} 2, \mathrm{~s} 3)$ \\
\hline 205 & S1 & S2 & S1 & S1 & & N1 & N1 & S1 & S1 & N1 & $\mathrm{N} 1(\mathrm{~s} 2, \mathrm{~s} 3, \mathrm{n})$ \\
\hline 211 & S1 & S1 & S1 & S1 & & S1 & S2 & S1 & S1 & S1 & S2(s3) \\
\hline 220 & N1 & S2 & S1 & S1 & & S1 & S1 & S1 & S1 & S2 & $\mathrm{N} 1(\mathrm{t}, \mathrm{s5})$ \\
\hline 223 & N1 & S2 & S1 & S2 & & N1 & S1 & S1 & S1 & S1 & $\mathrm{N} 1(\mathrm{t}, \mathrm{s} 2, \mathrm{~s} 5)$ \\
\hline \multicolumn{12}{|c|}{ Terraced } \\
\hline 71 & S1 & S1 & S1 & S2 & & S1 & \begin{tabular}{|l|} 
S1 \\
\end{tabular} & S2 & S1 & S1 & S3(s4) \\
\hline 86 & SC & S1 & S1 & S1 & & S1 & S1 & S1 & S1 & S1 & $\mathrm{SC}(\mathrm{t})$ \\
\hline 93 & S3 & S1 & S1 & S2 & & S1 & S1 & S1 & S1 & S1 & S3(t) \\
\hline 105 & S3 & S2 & S1 & S1 & & S1 & S1 & S1 & S1 & S1 & S3(t,s5) \\
\hline 106 & SC & S1 & S2 & S2 & & S1 & S1 & S1 & S1 & S1 & S3(t,s5) \\
\hline 110 & S3 & S1 & S1 & S1 & & S1 & \begin{tabular}{|l|} 
S1 \\
\end{tabular} & S2 & S2 & S1 & $\begin{array}{l}\mathrm{S} 3(\mathrm{t}, \mathrm{s} 5) \\
\end{array}$ \\
\hline 118 & SC & S1 & S1 & S1 & & S1 & S1 & S1 & S1 & N1 & $\mathrm{SC}(\mathrm{t}, \mathrm{s} 5, \mathrm{n})$ \\
\hline 129 & SC & S1 & S1 & S2 & & S1 & S3 & S2 & S2 & S1 & $\mathrm{SC}(\mathrm{t}, \mathrm{s} 5, \mathrm{~s} 3)$ \\
\hline 136 & S3 & S1 & S1 & S1 & & S1 & S1 & S1 & S1 & S1 & S3(t) \\
\hline 148 & S3 & S2 & S1 & S1 & & S1 & S1 & S1 & S1 & S1 & S3(t) \\
\hline 164 & N1 & S2 & S1 & S1 & & S1 & S1 & S2 & S3 & S1 & $\mathrm{N} 2(\mathrm{t}, \mathrm{s} 5)$ \\
\hline 175 & N1 & S2 & S1 & S1 & & S1 & \begin{tabular}{|l|} 
S1 \\
\end{tabular} & S1 & S1 & S1 & N1(t) \\
\hline \multicolumn{12}{|c|}{ Lava field and volcanic hills } \\
\hline 225 & N1 & S1 & S1 & S1 & & S1 & \begin{tabular}{|l|} 
S1 \\
\end{tabular} & $\mathrm{SC}$ & $\mathrm{SC}$ & S3 & $\mathrm{N} 1(\mathrm{t}, \mathrm{s} 5, \mathrm{n})$ \\
\hline 226 & N1 & S1 & S1 & S1 & & S1 & S1 & $\mathrm{SC}$ & S2 & S1 & $\mathrm{N} 1(\mathrm{t}, \mathrm{s} 4)$ \\
\hline \multicolumn{12}{|c|}{ Alluvial fan } \\
\hline 190 & S1 & S1 & S2 & S2 & & S1 & S1 & S1 & S1 & S1 & S2(s1) \\
\hline 197 & S1 & S2 & $\mathrm{SC}$ & S1 & & SC & S1 & S1 & S1 & S3 & $\mathrm{SC}(\mathrm{s} 2, \mathrm{n})$ \\
\hline 204 & S1 & S2 & $\mathrm{SC}$ & S1 & & S1 & N1 & S1 & S1 & N1 & $\mathrm{N} 1(\mathrm{~s} 3, \mathrm{n})$ \\
\hline 208 & S1 & S1 & S1 & $\mathrm{S} 2$ & & S1 & S1 & S1 & S1 & S1 & S2(s1) \\
\hline 218 & S1 & S1 & S1 & S1 & & S1 & S1 & S1 & S1 & S1 & S1 \\
\hline
\end{tabular}

t- Topography s2- Depth (s2'= for bedrock, s2 for water table) w- Drainage s3- CaCO3\%

$\mathrm{s} 1-$ Texture. $\left(\mathrm{s} 1^{\prime}=0-25 \mathrm{~cm}, \mathrm{~s} 1=>25 \mathrm{~cm}\right.$ depth) $\mathrm{s} 4-$ Coarse fragments $\left(\mathrm{s} 44^{\prime}=0-25 \mathrm{~cm}, \mathrm{~S} 4=>25 \mathrm{~cm}\right.$ depth$) \quad \mathrm{n}-\mathrm{Salinity} \&$ Alkalini 
Table (6):Total area and area of different suitability classes of irrigated agriculture and their ratios, according to FAO (1979 and 1983).

\begin{tabular}{|r|c|c|}
\hline \multicolumn{1}{|c|}{ Suitability classes } & Area (km 2) & \% Of the total area \\
\hline Highly suitable (S1) & 1059.1 & 13.0 \\
\hline Moderately suitable (S2) & 1372.2 & 16.8 \\
\hline Marginally suitable (S3) & 1287 & 15.8 \\
\hline Suitable with restriction (SC) & 2938.9 & 36.0 \\
\hline Currently not suitable (N1) & 1326.8 & 16.2 \\
\hline Permentally not suitable (N2) & 181.2 & 2.2 \\
\hline Total area & 8165.2 & 100.0 \\
\hline
\end{tabular}

mostly ascribed to the presence of one or two than four slight limitations and/or two or three moderate limitations. The land indice mostly situated between 50 and 75 . Most of these soils are characterized by flat surface, very deep, loam to clay loam textured, well drained, with optimum or nearly optimum infiltration rates $(0.8-7.0 \mathrm{~cm} / \mathrm{h})$ and free of salts. Agriculture limitations are mostly ascribed to the presence of one or two moderate limitations with regard to (Table 3):

- Texture is sandy loam as in some soils of wadis pediplain with deep soils and alluvial plain. Land indice varies between 61.8 and 75 , so subclass $\mathrm{S} 2_{(\mathrm{s} 1)}$ is suggested.

- Salinity is medium as in some soils of pediplain with deep soils. Land indice is 66.6 and subclass $\mathbf{S} 2_{(\mathrm{n})}$ is suggested.

- Infiltration rate is rather slow $(0.21-0.43 \mathrm{~cm} / \mathrm{h})$ as in some soils of pediplain with deep soils and alluvial plain. Land indice lies between 50.3 and 63 and subclass $S 2_{(\mathrm{s} 1, \mathrm{w})}$ is suggested.

- Marginally suitable land (S3)

Soils within this capability class have limitations which in aggregate are severe for sustained application of a given use and will so reduce productivity or benefits or increase required inputs. The land have more than $2 / 3$ moderate limitations and no more than 1 severe limitation that however does not exclude the use of the land. The land indice ranges between 25 and 50 . Soils within this capability class need special management that will increase largely inputs, in addition, choice for plants to be utilized and benefits expected are low. At the area under consideration, most of the studied soil profiles could be classified as marginally suitable including soils of Coastal plain, soils of wadis, soils of pediplain with deep soils, soils of alluvial plain, and soils of footslopes.

Agriculture limitations include moderate or severe limitations with regard to:

1- Salinity is severe as represented by the cultivated localities at the coastal plain and pediplain with deep soils.
2- Low available moisture capacity (texture is sand or loamy sand) as shown in soils of the coastal plain, wadis, pediplain with deep soils and the alluvial plain.

3- Rock fragments at surface are 37 and $76 \%$.

4- Topography is undulating or rolling as shown in soils of the coastal plain pediplain with deep soils and footslopes.

5- Carbonate content is severe $(64.8 \%)$ as shown in the (alluvial plain).

6- Infiltration rate is very rapid $(11-19.44 \mathrm{~cm} / \mathrm{h})$ as in soil profiles.

Based on these agriculture limitations the following subclasses could be defined, Table (3):

$S 3_{(\text {n) }}$ Represents the marginally suitable land for irrigated agriculture due to salinity severe limitation. It occupies the cultivated localities at the coastal plain. The capability index is 38.8 .

$\mathrm{S} 3_{(\mathrm{n}, \mathrm{s} 1, \mathrm{t})}$ Represents the soils have in addition to severe salinity, moderate limitations due to texture (SL) and topography (undulating). Land capability indice is 36 .

$\mathrm{S} 3_{(\mathrm{s} 1)}$ These soils have severe texture limitation (LS). Capability index varies between 42.8 and 50.

$\mathrm{S} 3_{(\mathrm{s} 1, \mathrm{t})}$ Represents soils have in addition to severe texture limitation (loamy sand), moderate limitation due to undulating topography as in soils of pediplain with deep soils and wadis. The land indice lies between 31.7 and 50.3

$\mathrm{S} 3_{(\mathrm{w}, \mathrm{s} 1)}$ Represents soils have severe limitation due to rapid infiltration rate and moderate texture as shown in some soil wadis and alluvial plain.The capability index ranges from 27 to 48.2

$\mathrm{S} 3_{(\mathrm{t}, \text { w) }}$ Represents the marginally suitable land which are characterized by rolling topography as severe limitation and rather slow infiltration rate $(0.43 \mathrm{~cm} / \mathrm{h})$ as moderate limitation (footslopes). The capability index is $\mathbf{4 3 . 2}$

$S 3_{(t, s 1, s 3)}$ Represents the marginally suitable land with moderate limitations due to undulating topography, moderately coarse textured (SL) 
and high $\mathrm{CaCO}_{3}$ content (64.8\%) (alluvial plain). The land capability indice is 50

$S 3_{(t, s 1, w)}$ Represents the marginally suitable land with moderate limitations due to topography (undulating), texture, and drainage (coastal plain, pediplain with deep soils and footslopes).

Soils characterized by severe salinity, rolling topography, subsurface calcic horizon require high input levels for reclamation and good management.

Currently unsuitable land (N1)

These are soils which may be surmountable in time, but which can't be corrected with existing knowledge at currently acceptable cost. Limitations are so severe to preclude successful stained use of the land in the given manner. Limitations of land within this class are so severe to increase largely development cost and decrease choice of plants and the benefits expected to be gained. Soil profiles are deep, sandy loam textured, highly affected with salts, and water table fluctuates between 90 to $130 \mathrm{~cm}$. Soil limitations are mainly ascribed with severe salinity and drainabilty. Suitable index less than 15 and subclass $\left(\mathrm{N}_{\mathrm{nw}}\right)$ is suggested for soils, Table (3). From economical point of view, these soils require high input levels for leaching salts and improving drainability. After good soil management these soils can be cultivated with highly salt tolerant crops.

\section{Acknowledgment}

The authors extend their thanks to King AbduIaziz City for Science and Technology (KACST) for financial support of project \# AT-23 51 under the title Assessment of land degradation in some irrigated soils (Causes and Features) in Saudi Arabia.

\section{REFERENCES}

Al-Malik A. S. (1994). Survey and Classification of Soils on A Selected Area of Wadi Hanifa Basin, Saudi Arabia. M. Sc. Thesis, Soil Science Department, College of Agriculture, King Saud University, Saudi Arabia.

Al-Sheikh A. A., El-Kady H. A., Al-Awajy M. H., Sallam A. Sh. and Al-Malik A. S. (1995). Clay minerals of soils at Wadi Hanifa (Najd Plateau, Saudi Arabia). Egypt. J. Appl. Sci., 10(6), pp. 411-426.

Al-Turki A. M., Al-Faraj A. S., Sallam A. Sh., Khalil J. A. and Al- Malik A. S., (2008). Survey of soil resources and water quality evaluation in Southern Tohama plains,
Saudi Arabia. King Abdul Aziz City for Sciences and technology, Riyadh.

Dent D. and Young A. (1981). Soil Survey and Land Evaluation. George Allen and Unwin, London.

FAO (1976). A framework for land evaluation. Food and Agriculture Organization of the United Nations, Soil Bulletin No. 32. Rome.

http://www.fao.org/docrep/x5310e/x5310e0 0. htm.

FAO (1978). Report on the Agro-ecological zones project. World Soil Resources Report 48. FAO, Rome.

FAO (1979). Yield response to water. J. Doorenbos and A.H. Kassam. FAO Irrigation and Drainage Paper 33. Rome, FAO.

FAO (1983). Guidelines: Land evaluation for rainfed agriculture. FAO Soils Bulletin, No. 52,FAO,Rome. http://www.fao.org/ag/AGL/agll/landuse/fa opubs.stm.

FAO (1984). Guidelines: Land evaluation for forestry. FAO Soils Bulletin, No. 48, Rome. http://www.fao.org/ag/AGL/agll/landuse/fa opubs.stm.

FAO (1983). Guidelines: Land Evaluation for Rainfed Agriculture Soils Bulletin No. 52 Rome: 237

FAO (1993). Guideline for Land Use Planning. FAO Development Series, No: 1, FAO, Rome, 96 pp.

Mashhady A. S., Hammad M. A. and Reda M. (1986). Soil Resources and Land Potential for Al-Qasseem Region, Saudi Arabia. Agric. Research Center, Coll. Agric. King Saud Univ.1-95.

Ministry of Agriculture and Water. (1985). General Soil Map of the Kingdom of Saudi Arabia. Prepared by Ministry of Agriculture and Water in Co-operation with Saudi Arabia-United States Joint Commission of Economic Co-operation. pp. 1-47.

Ministry of Agriculture and Water. (1988). Climate Atlas of Saudi Arabia. Prepared by Ministry of Agriculture and Water in Cooperation with Saudi Arabia-United States Joint Commission of Economic Cooperation. pp. 1-118.

Ministry of Agriculture and Water. (1995). The Land Resources. Ministry of Agriculture and Water, Land Management Dept., pp. 133.

Rahimi Lake H., Taghizadeh Mehrjardi R. 
Akbarzadeh A. and Ramezanpour $\mathrm{H}$. (2009). Qualitative and Quantitative Land Suitability Evaluation for Olive (Olea europaea L.) Production in Roodbar Region, Iran. Agricultural Journal, Volume: 4, Issue: 2, P 52-62.

Sys C. (1979). Evaluation of the physical environment for irrigation in terms of land characteristics and land qualities. World Soil Resources Reports, No. 50, FAO, Rome.

Sys C. (1993). Land Evaluation, Part III, Crop requirements. No. 7, General Administration for Development Cooperation Place du champ de Mar. 5 bte 57-1050 BrusselsBelgium.

Sys C. and Verheye W. (1978). An attempt to the evaluation of physical characteristic for irrigation according to FAO frame work for land evaluation, Inst. Train. Center Post.Gard. Soil state Univ. Ghent, Belgium.

Sys C., Ranst. V and Debaveye. J. (1991). Land Evaluation Part II, Method in Land evaluation Agricultural publication No. 7, ITC Ghent.

USBR (1951). Irrigated land use, Part 2: Land classification. Bureau of Reclamation Manual, Vol. 5. Washington: U.S. Government Printing Office.

$$
\begin{aligned}
& \text { تقييم الأر اضي لاستدامة الأنتاجية في سهول تهامة الجنوبية- المملكة العربية السعودية } \\
& \text { عبد الله بن سليمان الفراج - عبد العظيم شهوان سلام - على بن محمد التركي - الربل } \\
& \text { عبد الرحمن بن سعد المالك - جمال عبد الناصر خليل } \\
& \text { قسم علوم التزبة - كلية علوم الأغذية و الزر اعة - جامعة الملك سعود- المملكة العربية السعودية }
\end{aligned}
$$

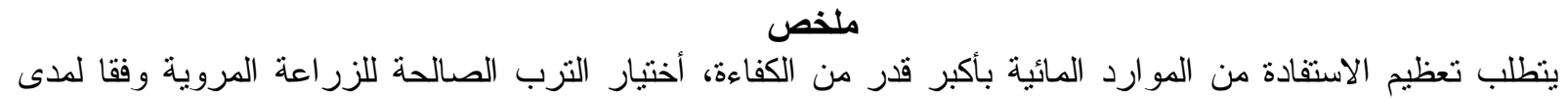

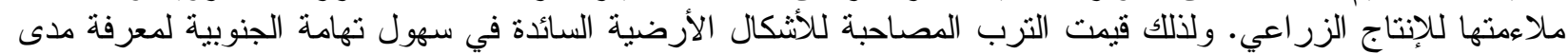

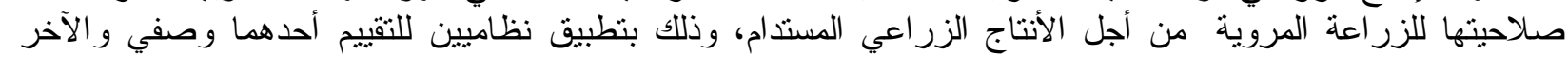

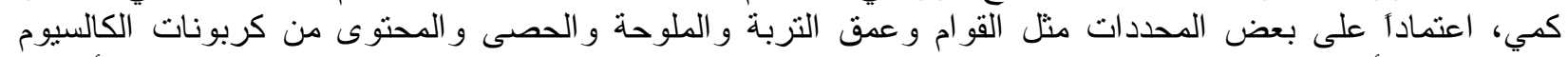

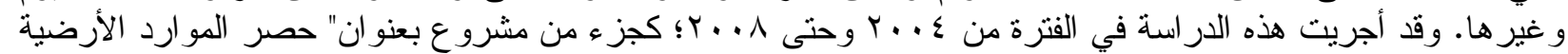

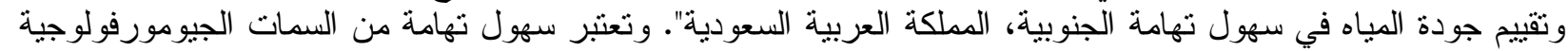

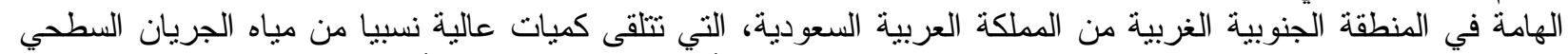

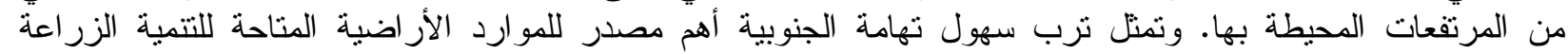

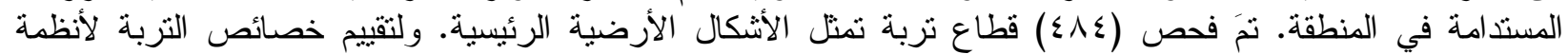

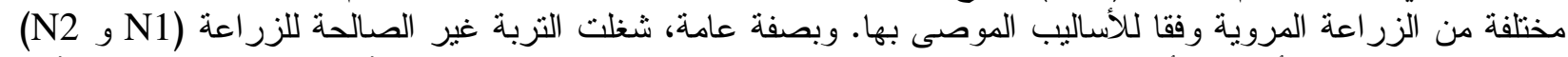

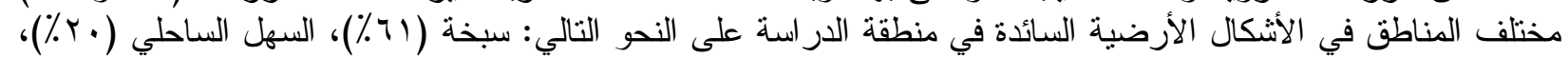

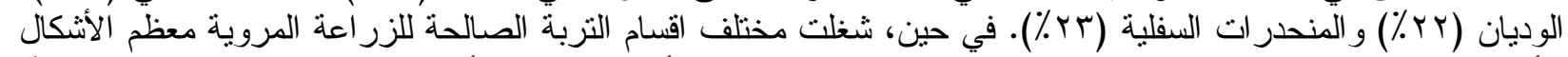

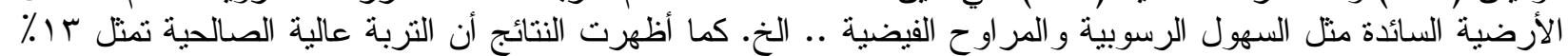

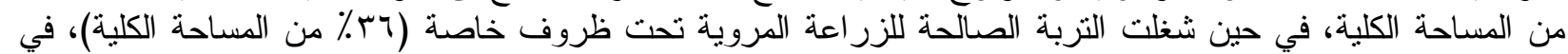

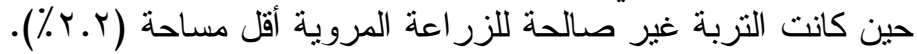

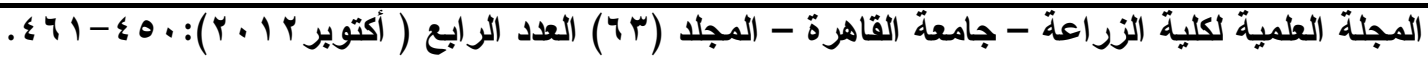

